\title{
Case Report \\ Trimethoprim-Sulfamethoxazole-Induced Hyperkalemia in a Patient with Normal Renal Function
}

\author{
L. Connor Nickels, ${ }^{1}$ Christine Jones, ${ }^{1}$ and Latha Ganti Stead ${ }^{2}$ \\ ${ }^{1}$ Department of Emergency Medicine, College of Medicine, University of Florida, 1329 SW 16th Street, P.O. Box 100186, \\ Gainesville, FL 32610-0186, USA \\ ${ }^{2}$ Division of Clinical Research, Department of Emergency Medicine \& Neurological Surgery and Toral Family Foundation, \\ Center for Brain Injury Research and Education, College of Medicine, University of Florida, 1329 SW 16th Street, P.O. Box 100186, \\ Gainesville, FL 32610-0186, USA
}

Correspondence should be addressed to Latha Ganti Stead, lstead@ufl.edu

Received 14 October 2012; Accepted 16 November 2012

Academic Editors: A. K. Exadaktylos, C.-C. Lai, and W. Mauritz

Copyright ( 92012 L. Connor Nickels et al. This is an open access article distributed under the Creative Commons Attribution License, which permits unrestricted use, distribution, and reproduction in any medium, provided the original work is properly cited.

The authors present a case of Trimethoprim-sulfamethoxazole-induced hyperkalemia in a patient with normal renal function. While toxicity of this drug has been reported in patients with renal insufficiency, this case highlights the toxicity associated with normal kidney function. Due to its popularity in the medical field and to the largely unrecognized effect of hyperkalemia, it is important to consider such adverse effects when prescribing TMX-SMX. One must be reminded of the possibility of the development of life-threatening hyperkalemia in relatively healthy patients.

\section{Introduction}

Trimethoprim-sulfamethoxazole (TMP-SMX) is a broadspectrum antibiotic used to treat a variety of infections. Due to its efficacy, its ease of dosing, and to the relatively low expense, it has become a popular choice among many physicians. However, as with any medications, adverse reactions may be experienced. Rash and gastrointestinal upset are the most commonly reported side effects associated with TMP-SMX. In contrast, hyperkalemia is rarely reported and most physicians are not aware of this potentially harmful side effect. The literature has long reported the occurrence of TMP-SMX-induced hyperkalemia in patients with acquired immunodeficiency syndrome (AIDS), patients with end stage renal disease (ESRD), and patients on high dose TMPSMX [1-11]. More recently, there have been reports of similar symptoms occurring in patients treated with standard dose TMP-SMX $[12,13]$, and in conjunction with other medications, such as enalapril and spironolactone [14-19]. We present a case of life-threatening TMP-SMX-induced hyperkalemia in a female with a normal creatinine whose only other identifiable risk factor was daily lisinopril.

\section{Case}

A 61-year-old female presented to the Emergency Department (ED) with a complaint of "I feel like I'm going to die." She reported being in her usual state of health until seven days prior when she developed cold symptoms. She was prescribed TMP-SMX for her upper respiratory tract infection and had completed four days of the antibiotic course at the time of her arrival in the ED.

Upon examination, the patient reported two days of progressively worsening weakness and fatigue and one day of chest pressure and shortness of breath. Prior to arrival, she experienced an acute increase in the generalized weakness, rendering her unable to ambulate without assistance. Also, she reported nausea and diaphoresis. She denied any additional accompanying symptoms.

The patient's past medical history was significant for diabetes, hypertension, lupus, and hypothyroidism. Her current medications were metformin, lisinopril, methotrexate, and levothyroxine. Her surgical and social histories were unremarkable and she was not aware of similar illnesses in her family. 
On physical exam, the patient was noted to be in extremis. Vital signs revealed a blood pressure of 190/65, a pulse of 100 , and respiratory rate greater than 20 . She was pale and diaphoretic in appearance. She was unable to sit upright on the stretcher without assistance or lift her extremities. Also, she was in moderate respiratory distress with tachypnea and increased work of breathing. Her breath sounds were coarse bilaterally. Cardiac exam was unremarkable for any pertinent findings other than tachycardia. On neurological exam, there was no focal deficit; however, there was significant generalized weakness throughout, 2/5 strength in all extremities. She was mentating normally.

Due to the patient's appearance, point of care (POC) testing was performed at the bedside. The results revealed a sodium level of 124 , a potassium level of 8.3 , a creatinine level of 1.0, a blood glucose of 361, and a troponin I level $<0.10$. The hemoglobin and hematocrit, as well as the venous blood gas, were all within the normal ranges. The patient's 12-lead electrocardiogram (EKG) showed a wide complex tachycardia with peaked $\mathrm{T}$ waves indicating hyperkalemic changes.

The hyperkalemia was treated immediately. The patient received calcium gluconate 1 gram IV, sodium bicarbonate 1 ampule IV, insulin 10 units IV, albuterol $2.5 \mathrm{mg} / 3 \mathrm{~mL}$ nebulized, and Kayexalate suspension $30 \mathrm{~g} / 120 \mathrm{~mL}$ PO. She was started on a continuous infusion of insulin for her hyperglycemia. Shortly after, she experienced marked improvement in symptoms. Her chest pressure, nausea, shortness of breath, and diaphoresis resolved. The patient was able to move all extremities and was noted to have 4/5 strength in all four. Her EKG began to normalize. Nephrology was contacted for emergent dialysis.

Approximately two hours later, the patient experienced a return of all her symptoms. Labs were rechecked, showing the same findings of hyperkalemia with a normal creatinine. The hyperkalemia protocol was repeated. A vascular catheter was placed in the patient's right femoral vein for emergent dialysis access. The patient was admitted for further care.

After arriving in the ICU, dialysis was performed, and her potassium level decreased to approximately 6. Overnight, the insulin infusion was continued. The next morning, the patient received another dose of Kayexalate. Throughout the day her potassium level trended downward and normalized between 4 and 5, and her EKG changes resolved. During her stay, the patient's creatinine remained stable, and her renal ultrasound revealed no abnormalities of the kidneys. She also had an echo performed that showed an ejection fraction of $60-65 \%$, and no wall motion abnormalities. It was determined that the patient's condition was due to TMPSMX-induced hyperkalemia in the setting of daily lisinopril.

\section{Discussion}

Hyperkalemia is a dangerous condition, potentially leading to cardiac dysrhythmias and death. It is most often linked to renal dysfunction, but may be seen with adrenal disease, with diseases which cause destruction of muscle tissue, or with medications. Some of the most widely used medications have been implicated, such as potassium supplements, potassium-sparing diuretics, angiotensin-converting enzyme inhibitors (ACE-I), angiotensin II receptor antagonists (ARB), trimethoprim-sulfamethoxazole, and heparin [14, 20]. We focus on one drug in particular, TMP-SMX.

Some of the earliest cases of trimethoprim-induced hyperkalemia were reported in patients with HIV/AIDS. These patients are typically treated with large doses of TMXSMX for Pneumocystis carinii [1-11]. It has been postulated that perhaps the declining renal function associated with the natural progression of the disease, combined with "highdose" TMX-SMX is responsible for the untoward effect, as renal dysfunction accelerates the incidence of hyperkalemia [21]. However, more recent clinical studies have shown that hyperkalemia can be seen even with "standard-dose" TMXSMX [15, 21-24].

One study in particular evaluated this relationship in immunocompetent patients in the outpatient setting [19]. This study looked at two groups: a treatment group and a control group. The treatment group was comprised of patients receiving "standard-dose" TMX-SMX which was defined as trimethoprim, $320 \mathrm{mg} /$ day, and sulfamethoxazole, $1,600 \mathrm{mg} /$ day. The control group included patients taking other antibiotics. The authors noted that the treatment group showed a statistically significant $(P<0.001)$ rise in potassium levels after 5 days of treatment. However, they reported only $6 \%$ of patients ( 3 patients) developed "severe" hyperkalemia, defined as $\geq 5.5$, and 0 patients developed clinically significant hyperkalemia [19]. This led to the conclusion that life-threatening hyperkalemia occurs much less frequently in outpatients treated with "standard-dose" TMX-SMX than in patients with AIDS or renal insufficiency, who may be prescribed a "high-dose" therapy [19].

However infrequent the occurrence, it is important to understand the mechanism by which the adverse effect occurs. Trimethoprim is structurally similar to the potassium-sparing diuretic amiloride [20, 24]. It competitively inhibits the sodium channels of the epithelium in the distal nephron, thereby impairing renal potassium excretion [24]. This alone may induce hyperkalemia, but one must consider that the causes of hyperkalemia may be additive [14]. For example, studies have shown that TMX-SMX in combination with ACE-Is significantly increases the risk of hospitalization secondary to hyperkalemia [18]. This is likely due to the suppression of angiotensin II, which leads to a decrease in aldosterone levels. Since aldosterone is responsible for increasing the excretion of potassium, ACE inhibitors ultimately cause retention of potassium.

Due to its popularity in the medical field and to the largely unrecognized effect of hyperkalemia, it is important to consider such adverse effects when prescribing TMXSMX. One must be reminded of the possibility of the development of life-threatening hyperkalemia in relatively healthy patients.

\section{Conflict of Interests}

The authors confirm that they have no conflict of interests or financial disclosures. 


\section{References}

[1] M. T. Sheehan and S. F. Wen, "Hyperkalemic renal tubular acidosis induced by trimethoprim/sulfamethoxazole in an AIDS patient," Clinical Nephrology, vol. 50, no. 3, pp. 188-193, 1998.

[2] H. Klinker, P. Langmann, M. Zilly, and E. Richter, "Drug monitoring during the treatment of AIDS-associated Pneumocystis carinii pneumonia with trimethoprim-sulfamethoxazole," Journal of Clinical Pharmacy and Therapeutics, vol. 23, no. 2, pp. 149-154, 1998.

[3] M. C. Porras, J. N. Lecumberri, J. L. P. Castrillon, M. C. Porras, and M. Larouche, "Trimethoprim/sulfamethoxazole and metabolic acidosis in HIV-infected patients," Annals of Pharmacotherapy, vol. 32, no. 2, pp. 185-189, 1998.

[4] H. J. Anders, J. R. Bogner, and F. D. Goebel, "Mild rhabdomyolysis after high-dose trimethoprim-sulfamethoxazole in a patient with HIV infection," European Journal of Medical Research, vol. 2, no. 5, pp. 198-200, 1997.

[5] O. Giraud, F. Thomas, F. Aussavy, J. J. Jupas, and Y. Ravaud, "Hyperkaliemia and acute renal failure after overdose of trimethoprim-sulfamethoxazole in one AIDS patient," Annales de Medecine Interne, vol. 148, no. 2, pp. 185-186, 1997.

[6] L. B. Mihm, R. C. Rathbun, and B. H. Resman-Targoff, "Hyperkalemia associated with high-dose trimethoprim-sulfamethoxazole in a patient with the acquired immunodeficiency syndrome," Pharmacotherapy, vol. 15, no. 6 I, pp. $793-$ 797, 1995.

[7] P. Domingo, S. Ferrer, J. Cruz, R. Morla, and J. Ris, "Trimethoprim-sulfamethoxazole-induced renal tubular acidosis in a patient with AIDS," Clinical Infectious Diseases, vol. 20, no. 5, pp. 1435-1437, 1995.

[8] M. Monteagudo, J. Sola, J. Mestre, and D. Conesa, "Hyperkalemia during therapy of Pneumocystis carinii pneumonia with trimethoprim-sulfamethoxazole," Revista Clinica Espanola, vol. 195, no. 3, pp. 198-199, 1995.

[9] H. Noto, Y. Kaneko, T. Takano, and K. Kurokawa, "Severe hyponatremia and hyperkalemia induced by trimethoprimsulfamethoxazole in patients with Pneumocystis carinii pneumonia," Internal Medicine, vol. 34, no. 2, pp. 96-99, 1995.

[10] M. J. Choi, P. C. Fernandez, A. Patnaik et al., "Brief report: trimethoprim-induced hyperkalemia in a patient with AIDS," The New England Journal of Medicine, vol. 328, no. 10, pp. 703 706, 1993.

[11] M. Koç, A. Bihorac, C. I. Ozener, G. Kantarci, and E. Akoglu, "Severe hyperkalemia in two renal transplant recipients treated with standard dose of trimethoprim-sulfamethoxazole," American Journal of Kidney Diseases, vol. 36, no. 3, article E18, 2000.

[12] E. P. Perlmutter, D. Sweeney, G. Herskovits, and M. Kleiner, "Case report: severe hyperkalemia in a geriatric patient receiving standard doses of trimethoprim-sulfamethoxazole," American Journal of the Medical Sciences, vol. 311, no. 2, pp. 84-85, 1996.

[13] M. A. Marinella, "Case report: reversible hyperkalemia associated with trimethoprim-sulfamethoxazole," American Journal of the Medical Sciences, vol. 310, no. 3, pp. 115-117, 1995.

[14] H. Velazquez, M. A. Perazella, F. S. Wright, and D. H. Ellison, "Renal mechanism of trimethoprim-induced hyperkalemia," Annals of Internal Medicine, vol. 119, no. 4, pp. 296-301, 1993.

[15] M. A. Marinella, "Trimethoprim-induced hyperkalemia: an analysis of reported cases," Gerontology, vol. 45, no. 4, pp. 209 212, 1999.

[16] P. Noize, H. Bagheri, G. Durrieu et al., "Life-threatening drug-associated hyperkalemia: a retrospective study from laboratory signals," Pharmacoepidemiology and Drug Safety, vol. 20, no. 7, pp. 747-753, 2011.

[17] J. F. Bugge, "Severe hyperkalaemia induced by trimethoprim in combination with an angiotensin-converting enzyme inhibitor in a patient with transplanted lungs," Journal of Internal Medicine, vol. 240, no. 4, pp. 249-251, 1996.

[18] T. Antoniou, T. Gomes, D. N. Juurlink, M. R. Loutfy, R. H. Glazier, and M. M. Mamdani, "Trimethoprim-sulfamethoxazole-induced hyperkalemia in patients receiving inhibitors of the renin-angiotensin system: a population-based study," Archives of Internal Medicine, vol. 170, no. 12, pp. 1045-1049, 2010.

[19] R. Alappan, G. K. Buller, and M. A. Perazella, "Trimethoprimsulfamethoxazole therapy in outpatients: is hyperkalemia a significant problem?" American Journal of Nephrology, vol. 19, no. 3, pp. 389-394, 1999.

[20] M. A. Perazella, "Trimethoprim is a potassium-sparing diuretic like amiloride and causes hyperkalemia in high-risk patients," American Journal of Therapeutics, vol. 4, no. 9-10, pp. 343-348, 1997.

[21] H. Mori, Y. Kuroda, S. Imamura et al., "Hyponatremia and/or hyperkalemia in patients treated with the standard dose of trimethoprim-sulfamethoxazole," Internal Medicine, vol. 42, no. 8, pp. 665-669, 2003.

[22] M. A. Perazella and R. L. Mahnensmith, "Trimethoprim-sulfamethoxazole: hyperkalemia is an important complication regardless of dose," Clinical Nephrology, vol. 46, no. 3, pp. 187192, 1996.

[23] M. A. Perazella, "Hyperkalemia and trimethoprim-sulfamethoxazole: a new problem emerges 25 years later," Connecticut Medicine, vol. 61, no. 8, pp. 451-458, 1997.

[24] M. A. Perazella, "Trimethoprim-induced hyperkalaemia. Clinical data, mechanism, prevention and management," Drug Safety, vol. 22, no. 3, pp. 227-236, 2000. 


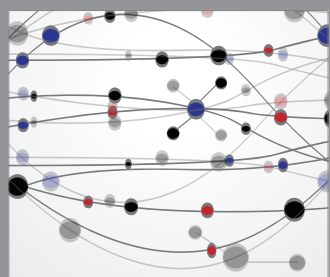

The Scientific World Journal
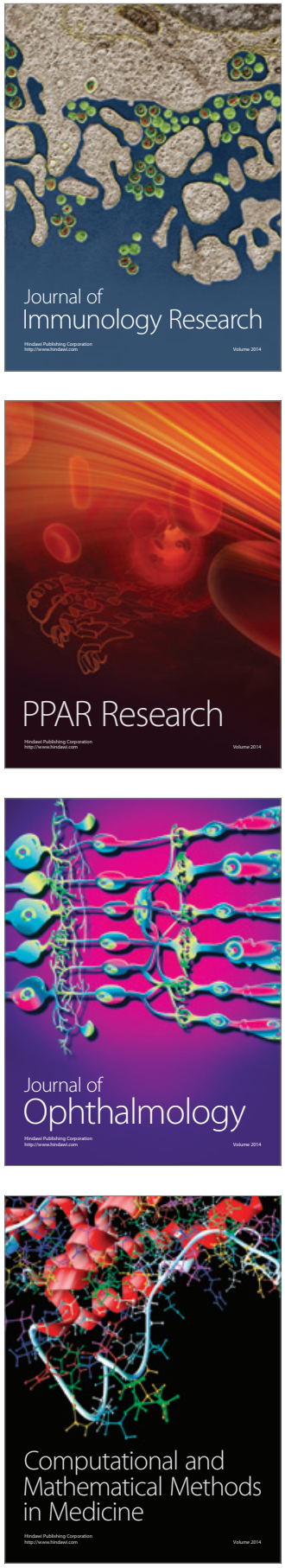

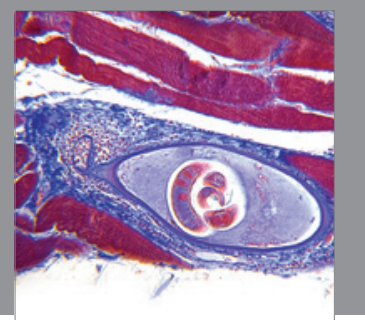

Gastroenterology

Research and Practice
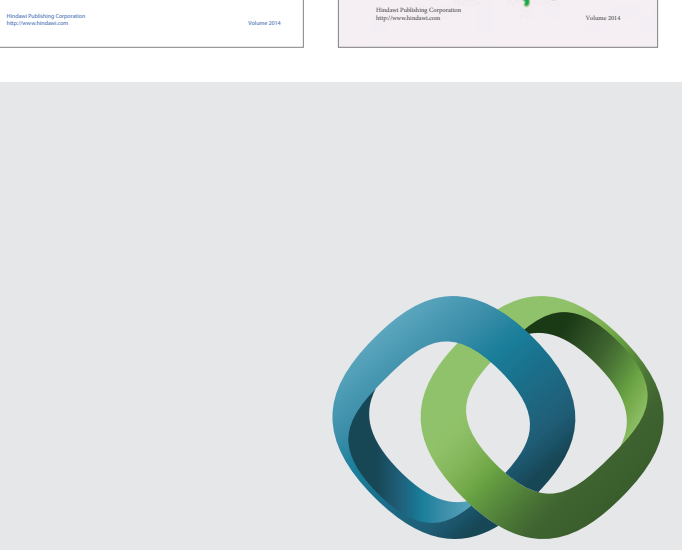

\section{Hindawi}

Submit your manuscripts at

http://www.hindawi.com
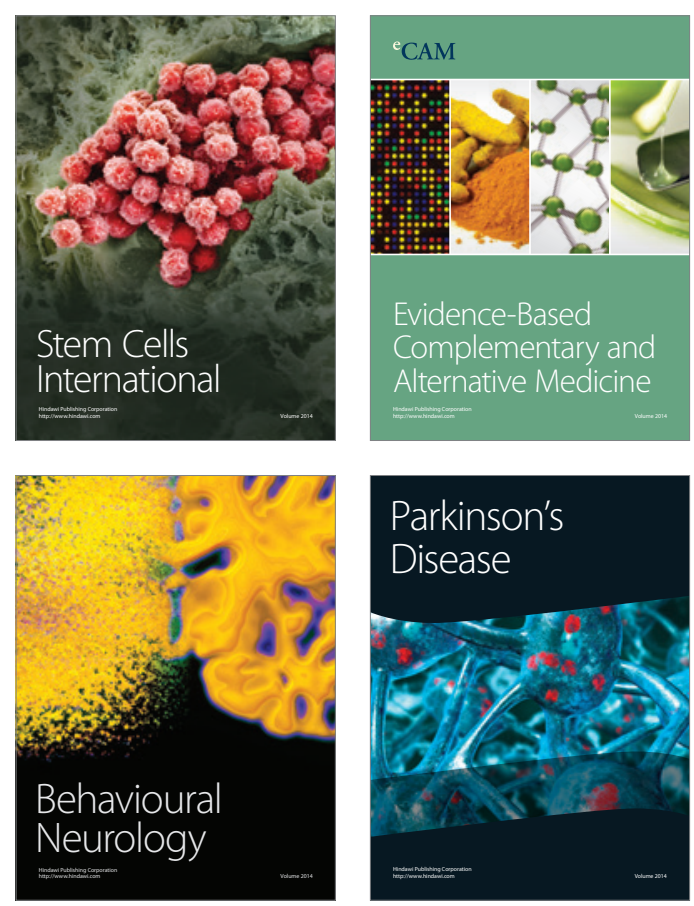

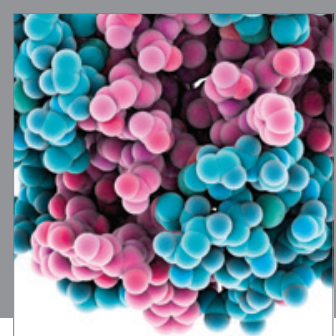

Journal of
Diabetes Research

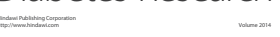

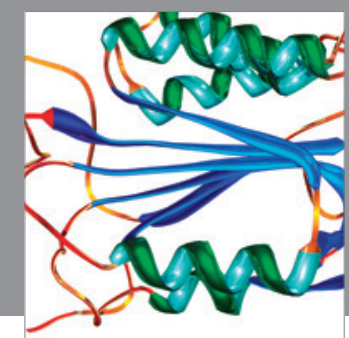

Disease Markers
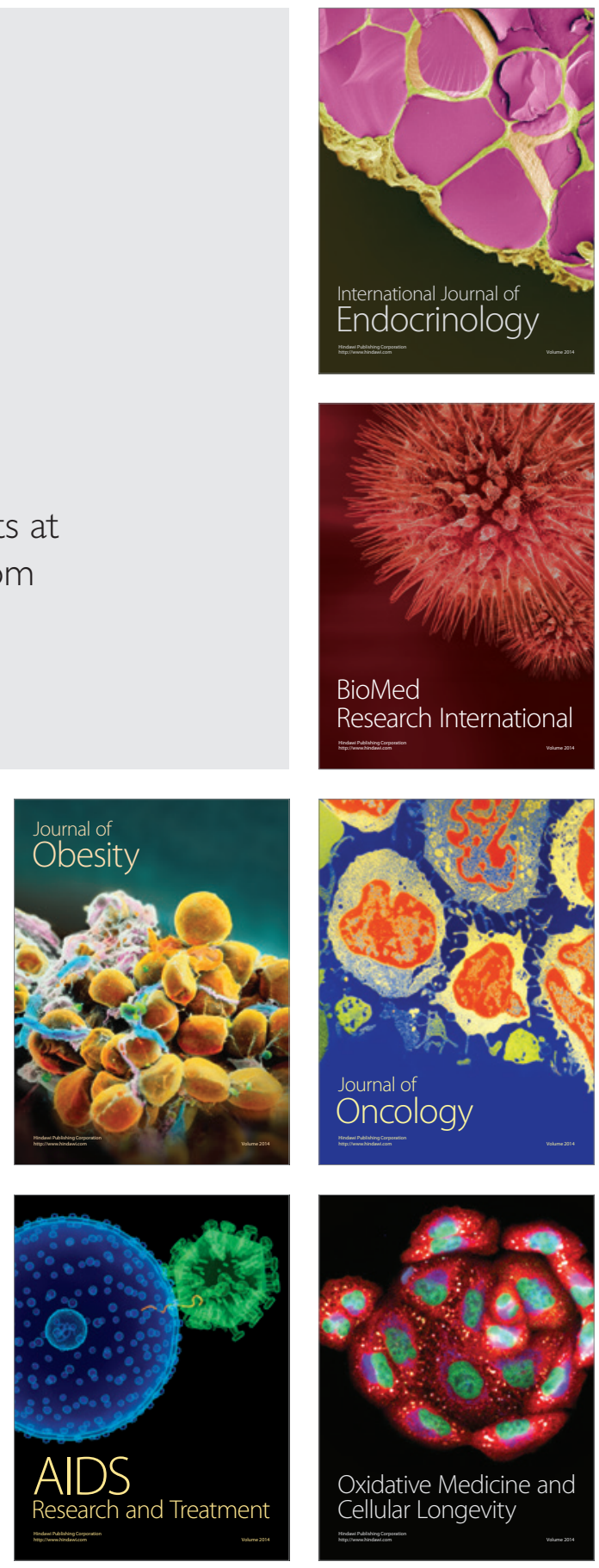